\title{
Long-erm complications in men who have early or late radiotherapy after radical prostatectomy
}

\author{
Robert J. Sowerby, MD; Johan Gani, MD; ${ }^{\dagger}$ Harold Yim, MD; ${ }^{*}$ Sidney B. Radomski, MD, FRCSC; \\ Charles Catton, MD, FRCPC ${ }^{\S}$
}

*Division of Urology, Department of Surgery, Toronto Western Hospital and University of Toronto, Toronto, ON; †Department of Surgery, Austin Health, Victoria, Australia; §Radiation Medicine Program, Princess Margaret Hospital and University of Toronto, Toronto, ON

Cite as: Can Urol Assoc J 2014;8(7-8):253-8. http://dx.doi.org/10.5489/cuaj.1764 Published online August 11, 2014.

\section{Abstract}

Introduction: Choosing adjuvant radiotherapy (RT) or salvage RT after radical prostatectomy (RP) for locally advanced prostate cancer is controversial. Performing RT early after RP may increase the risk of urinary complications compared to RT performed later. We evaluated the urinary complication rates of men treated with surgery followed by early or late RT.

Methods: Using a retrospective chart review, we compared rates of urinary incontinence (UI), bladder neck contracture (BNC), or urethral stricture in men with prostate cancer treated with early RT $(<6$ months after RP) or late RT ( $\geq 6$ months after RP), 3 years after RT. Results: In total, 652 patients (between 2000 and 2007) underwent early RT $(162,24.8 \%)$ or late RT $(490,75.2 \%)$ after RP. The mean time to early RT was 3.6 months (range: 1-5 months) and to late RT was 30.1 months (range: 6-171 months). At 3 years post-RT, UI rates were similar in the early RT and the late RT groups $(24.5 \% \mathrm{vs}$. $23.3 \%$, respectively, $p=0.79$ ). Prior to $\mathrm{RT}, 27 / 652(4 \%)$ patients had a BNC and 11/652 (1.7\%) had a urethral stricture, of which only 1 BNC persisted at 3 years post-RT. After RT, 17/652 (2.6\%) BNC and 4/652 (0.6\%) urethral stricture developed; of these, 6 $\mathrm{BNC}$ and 2 urethral strictures persisted at 3 years.

Conclusion: Rates of UI, BNC, and urethral stricture were similar with early and late RT at 3 years post-RT. These findings suggest that the timing of RT after RP does not alter the incidences of these urinary complications and can aid in the decision-making process regarding adjuvant RT versus salvage RT.

\section{Introduction}

Radiotherapy (RT) after radical prostatectomy (RP) is an effective treatment option for locally advanced prostate cancer (T3 or positive surgical margins). ${ }^{1-6}$ Phase III studies have shown the benefit of adjuvant RT over a wait-and-see strategy. ${ }^{2,4,5}$ Salvage RT, performed later and triggered by biochemical recurrence, has been shown to be beneficial in several retrospective analyses. ${ }^{6-9}$ Although the results of retrospective trials comparing adjuvant and salvage RT favour adjuvant $\mathrm{RT}$, , $^{10-13}$ no prospective randomized trials have been completed. The timing of postoperative RT remains controversial and treatment toxicity should be one of the factors considered. ${ }^{14,15}$

Adjuvant RT is typically delayed for a couple of months after RP to allow for healing, as these patients are at increased risk of urinary incontinence (UI), bladder neck contracture (BNC) and urethral stricture. ${ }^{16}$ Adjuvant RT has shown worse genitourinary (GU) toxicity as compared to wait-and-see treatment strategy with rates of $\mathrm{UI}$ and urethral stricture between $6 \%$ to $20 \%$ and $6 \%$ to $18 \%$, respectively. ${ }^{3,17}$ Toxicity and long-term complication rates for salvage RT are similar to those of adjuvant RT; however, the effect of timing of postoperative RT on long-term complication rates is unknown and comparison of complication rates associated with early or late RT have not been previously reported. In this study, we evaluated the complication rates of men treated with RP followed by early or late RT.

\section{Methods}

We performed a retrospective analysis of patients at our institution (University Health Network, Toronto, Canada) who underwent RT after RP for prostate cancer, with permission from the hospital research ethics board. We included men who had RT at our institution after RP for prostate cancer, with at least a 3-year follow-up. These patients were divided into early RT ( $<6$ months after surgery) or late RT ( $\geq 6$ months after surgery) groups. Using a retrospective chart review, we recorded patient demographics, treatment characteristics and complications from RT. Data collected were based on routine clinical follow-up and divided into year 1, year 2 and year 3 after RT. Clinical follow-up varied based on the pathologic stage, patient factors, clinician routine and prostate-specific antigen (PSA). On every clinic visit after surgery, patients were asked by their clinician 
Sowerby et al.

about voiding function, incontinence and erectile function. Their responses were recorded in the clinical notes. UI was defined as the presence of any reported leakage. If UI status could not be determined, patients were marked as unknown and excluded from the analysis. BNC and urethral stricture status were based on cystoscopic findings, with the need for cystoscopy based on lower urinary tract symptoms. For BNC and urethral stricture, patients were marked as unknown and excluded from the analysis if they previously had BNC or urethral stricture and their status could not be determined. We compared the complication rates in each group and time interval using Fisher's Exact t-test.

\section{Results}

A retrospective chart review revealed 652 patients with 3 years of follow-up who underwent RT after RP between January 2000 and October 2007. Of these patients, 162 underwent early RT (24.8\%) and 490 underwent late RT (75.2\%). The mean time to RT was 3.6 months (range: $1-5$ months) in the early RT group and 30.1 months (range: 6-171 months) in the late RT group (Table 1).

In the early and late RT groups, we tallied the number of patients per year with UI that developed postoperatively before RT (pre-existing) or after RT (new-onset) (Fig. 1). UI developed postoperatively but before RT in 88 patients $(54.3 \%)$ in the early RT group and in 170 patients $(34.7 \%)$ in the late RT group. Including both pre-existing and new-onset $\mathrm{UI}$, at 3 years post-RT, 27 patients $(24.5 \%)$ in the early RT group and 72 patients $(23.3 \%)$ in the late RT group had UI $(p=0.79)$ (Table 2). Twelve patients ( 5 in the early RT group and 7 in the late RT group) received an artificial urinary sphincter (AUS) during the first 3 years after RT $(p=0.18)$.

In both groups, 27 patients ( $4.1 \%$ ) developed BNC postoperatively but before RT. Of these 27 pre-existing BNC, at 3 years after RT, 3 patients $(1.9 \%)$ were in the early RT group ( 2 cases were resolved and 1 was unknown) and

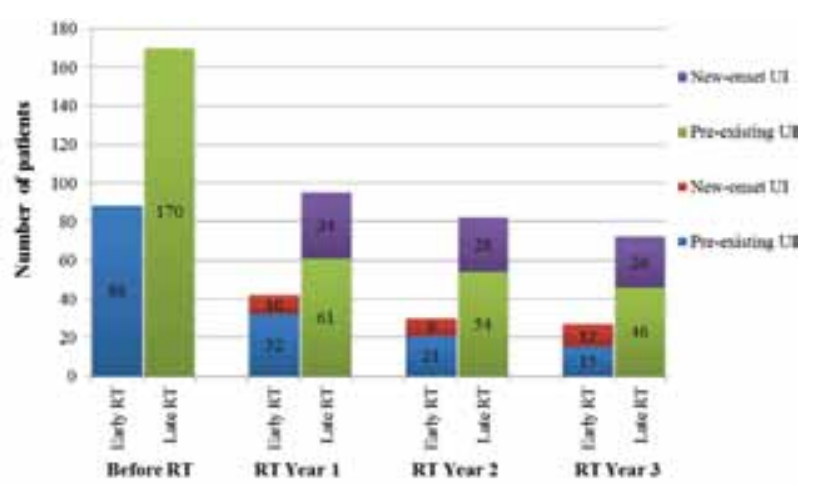

Fig. 1. Number of patients with UI, per-year, who developed before (preexisting) or after (new-onset) RT in the early and late RT groups. RT: radiotherapy; UI: urinary incontinence.
Table 1. Patient and treatment characteristics

\begin{tabular}{|c|c|c|c|c|}
\hline & \multicolumn{2}{|c|}{ Early RT } & \multicolumn{2}{|c|}{ Late RT } \\
\hline No. patients & \multicolumn{2}{|c|}{$162(24.8 \%)$} & \multicolumn{2}{|c|}{$490(75.2 \%)$} \\
\hline \multicolumn{5}{|l|}{ Age at RP } \\
\hline Mean & \multicolumn{2}{|c|}{60.47} & \multicolumn{2}{|c|}{60.44} \\
\hline Range & \multicolumn{2}{|c|}{$45-75$} & \multicolumn{2}{|c|}{$41-76$} \\
\hline \multicolumn{5}{|l|}{ Time to RT } \\
\hline Mean & \multicolumn{2}{|c|}{3.6 months } & \multicolumn{2}{|c|}{30.1 months } \\
\hline Range & \multicolumn{2}{|c|}{ 1-5 months } & \multicolumn{2}{|c|}{ 6-171 months } \\
\hline \multicolumn{5}{|l|}{ Radiation dose } \\
\hline Mean & \multicolumn{2}{|c|}{$66 \mathrm{~Gy}$} & \multicolumn{2}{|c|}{$66 \mathrm{~Gy}$} \\
\hline Range & \multicolumn{2}{|c|}{ 50-70 Gy } & \multicolumn{2}{|c|}{ 50-70 Gy } \\
\hline \multicolumn{5}{|l|}{ Types of RT } \\
\hline 3D conformal & \multicolumn{2}{|c|}{$141(87.0 \%)$} & \multicolumn{2}{|c|}{$415(84.7 \%)$} \\
\hline IMRT & \multicolumn{2}{|c|}{$21(13.0 \%)$} & \multicolumn{2}{|c|}{$75(15.3 \%)$} \\
\hline \multicolumn{5}{|c|}{ Nerve-sparing status } \\
\hline Unilateral & \multicolumn{2}{|c|}{$18(11.1 \%)$} & \multicolumn{2}{|c|}{$51(10.4 \%)$} \\
\hline Bilateral & \multicolumn{2}{|c|}{$32(19.8 \%)$} & \multicolumn{2}{|c|}{$90(18.4 \%)$} \\
\hline Non-sparing & \multicolumn{2}{|c|}{$18(11.1 \%)$} & \multicolumn{2}{|c|}{$40(8.2 \%)$} \\
\hline \multirow[t]{2}{*}{ Unknown } & \multicolumn{2}{|c|}{$94(58.0 \%)$} & \multicolumn{2}{|c|}{$309(63.0 \%)$} \\
\hline & Pre-RT & Post-RT & Pre-RT & Post-RT \\
\hline ADT & $\begin{array}{c}38 \\
(23.5 \%)\end{array}$ & $\begin{array}{l}50 \\
(30.9 \%)\end{array}$ & $\begin{array}{c}104 \\
(21.2 \%)\end{array}$ & $\begin{array}{l}142 \\
(29.0 \%)\end{array}$ \\
\hline
\end{tabular}

RT: radiotherapy; RP: radical prostatectomy; ADT: androgen deprivation therapy; IMRT: intensity-modulated radiotherapy.

24 patients $(4.9 \%)$ were in the late RT group (I case persisted and 3 were unknown). After RT, 17 patients (2.6\%) developed new-onset BNC; of these, 10 patients $(6.1 \%)$ were in the early RT group (4 cases persisted at 3 years after RT and 1 unknown) and 7 patients (1.4\%) in the late RT group (2 cases persisted at 3 years after RT (Fig. 2). Including both pre-existing and new-onset BNC, at 3 years post-RT, 4 persisted in the early RT group $(2.5 \%)$ and 3 persisted in the late RT group $(0.41 \%)(p=0.068)$.

Urethral stricture developed postoperatively in 11 patients $(1.7 \%)$ before RT, including 4 patients $(2.4 \%)$ in the early RT group and 7 patients $(1.4 \%)$ in the late RT group. At 3 years post-RT, none of these pre-existing urethral strictures persisted (4 were unknown). After RT, 4 patients (0.6\%) developed new-onset urethral stricture; of these, 1 patient $(0.62 \%)$ in the early RT group and 3 patients $(0.61 \%)$ in the late RT group. At 3 years post-RT, 1 urethral stricture persisted in the early RT group $(0.63 \%)$ and 1 urethral stricture persisted in the late RT group $(0.20 \%)(p=0.44)$ (Fig. 3 ).

\section{Discussion}

Phase III studies that compared adjuvant RT with a wait-andsee strategy have shown that adjuvant RT improved local control, ${ }^{2}$ biochemical progression-free survival, ${ }^{2,5}$ metastases-free survival ${ }^{4}$ and overall survival. ${ }^{4}$ Salvage RT has been shown to be beneficial in several retrospective analyses..$^{7-9}$ 


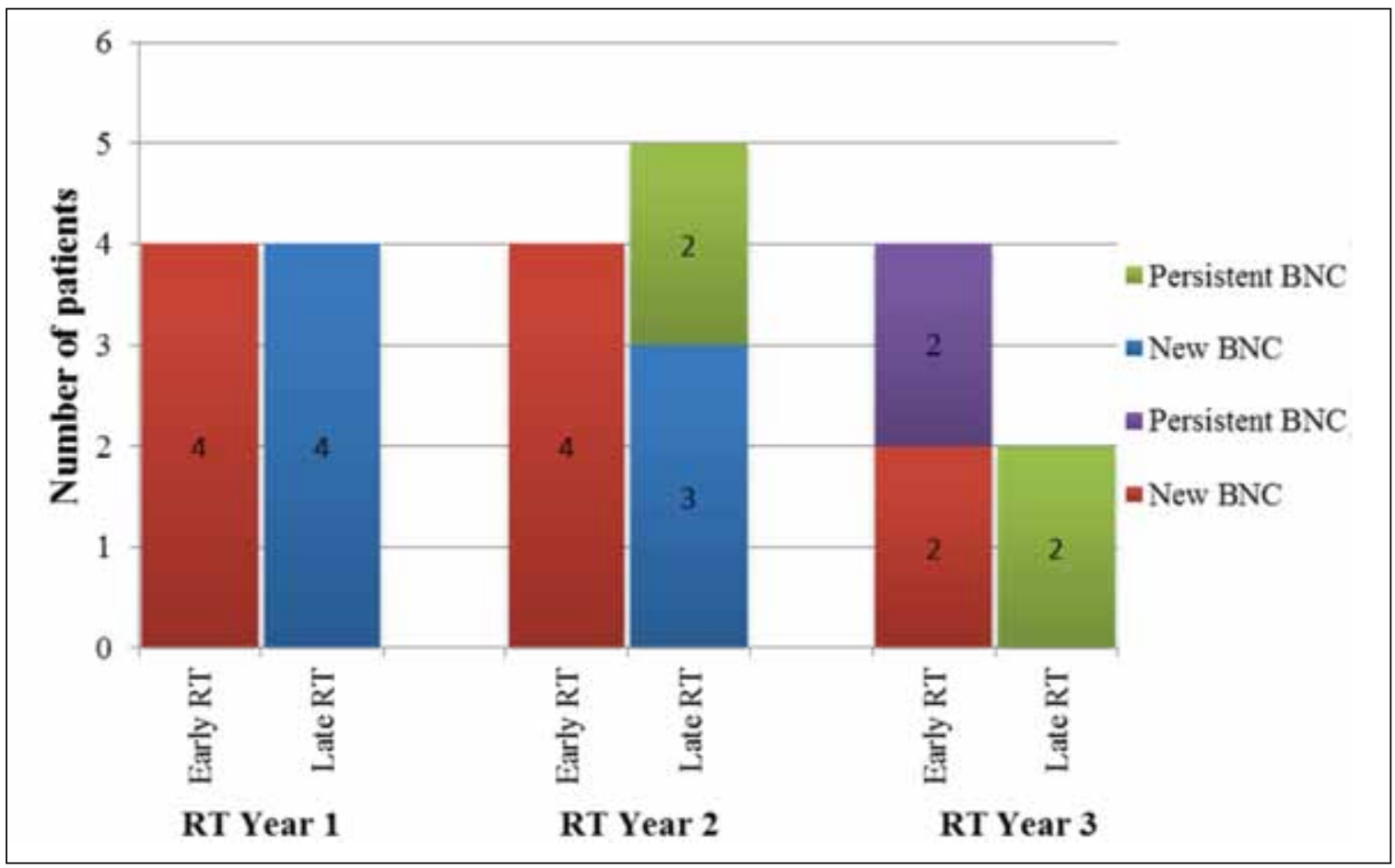

Fig. 2. Number of patients with BNC that developed post-radiotherapy (new and persistent) in the early and late RT groups. RT: radiotherapy; BNC: bladder neck contracture.

Although adjuvant RT and salvage RT have been compared in numerous retrospective studies with results favouring adjuvant RT, no prospective randomized trials have been completed..$^{10-13}$ In one study, adjuvant RT was only found to be beneficial over salvage RT if the side effects were negligible, emphasizing the importance of side effects on treatment timing. ${ }^{18}$ In addition, long-term follow-up from the European Organization for Research and Treatment of Cancer (EORTC) trial 22911 has shown that clinical progression-free survival rates previously reported with adjuvant RT were no longer clinically significant; moreover, in patients over 70, adjuvant RT had a detrimental effect on progression-free and overall survival. ${ }^{2}$ The decision to treat with adjuvant or salvage RT is perhaps more controversial than ever and should include patient preference, demographics, pathologic features and risk of complications.

RT causes capillary destruction with reduced vascularity and tissue fibrosis, relative tissue hypoxia and reduced nutrient supply. ${ }^{16}$ After RP, as tissues are healing, early RT may further increase the risk of urinary complications, including urethral stricture, BNC and UI, compared to RT performed later. As such, adjuvant RT is frequently delayed after RP to allow for wound healing and return of urinary continence..$^{19}$ In this study, the mean time to RT in the early group was 3.6 months with a range of 1-5 months indicating that some patients received RT earlier than recommended. Patients who received early RT in our series either opted for adjuvant
RT or exhibited early biochemical relapse, whereas those who received late RT generally exhibited late relapse or a relapse with a slow doubling time. Six months was chosen as a separation point between patients who received RT

\begin{tabular}{lccc}
\hline \multicolumn{4}{c}{ Table 2. Urinary incontinence rates per year } \\
& $\begin{array}{c}\text { Early RT, no. } \\
\text { (\%) }\end{array}$ & $\begin{array}{c}\text { Late RT, no. } \\
\text { (\%) }\end{array}$ & $\begin{array}{c}\text { Fisher's exact } \\
\text { t-test }\end{array}$ \\
\hline $\begin{array}{l}\text { No. patients } \\
\text { Urinary }\end{array}$ & 162 & 490 & \\
continence & & & \\
Leak & $88(54.3)$ & $170(34.7)$ & \\
No leak & 74 & 320 & \\
Unknown & 0 & 0 & $p=0.068$ \\
1 Year post-RT & & & \\
Leak & $42(30.2)$ & $95(22.4)$ & \\
No leak & 97 & 329 & \\
Unknown & 23 & 66 & \\
2 Years post-RT & & & \\
Leak & $30(24.6)$ & $82(22.2)$ & \\
No leak & 92 & 287 & \\
Unknown & 40 & 121 & \\
3 Years post-RT & & & \\
Leak & $27(24.5)$ & $72(23.3)$ & \\
No leak & 83 & 237 & \\
Unknown & 52 & 181 & \\
\hline RT: radiotherapy. & & & \\
\hline
\end{tabular}


Sowerby et al.

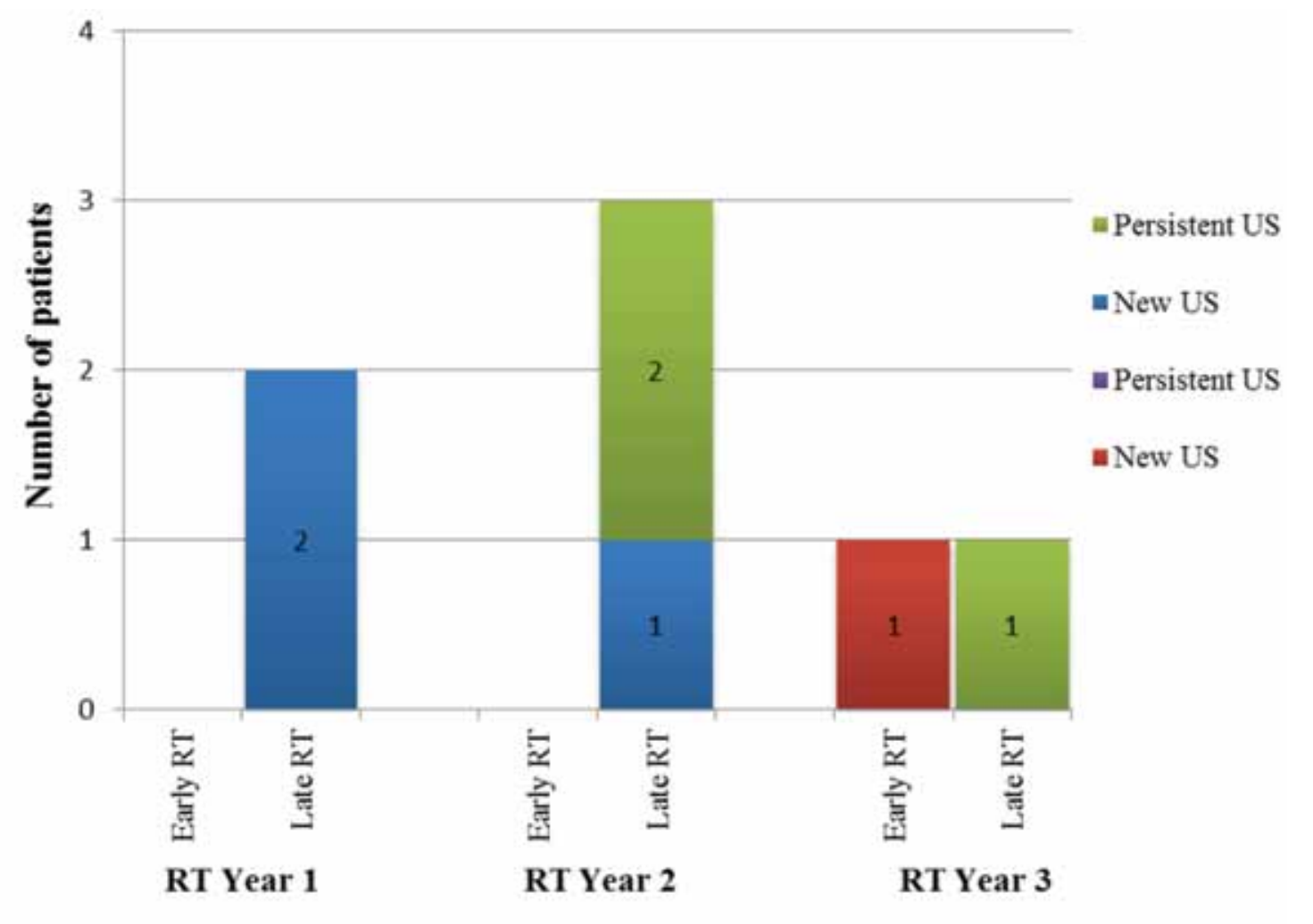

Fig. 3. Number of patients with US who developed post-RT (new and persistent) in the early and late RT groups. RT: radiotherapy; US: urethral stricture.

while potentially still healing and those who had RT when fully healed postoperatively, although most patients who received late treatment were well beyond the 6-month interval. Salvage RT for biochemical failure is typically administered before PSA increases above 0.2 to $0.5 \mathrm{ng} / \mathrm{mL}$ and the average time to salvage RT is reported between 12 and 24 months. 3,8,10,14,20,21 In this study, the time to RT in the late RT group was later at 30.1 months.

Chronic RT toxicity can present late after treatment, however most bladder complications manifest within 3 years of therapy.22 Data were collected in time intervals of 1,2 and 3 years after RT to capture most long-term complications. We observed new-onset of UI in 3 years post-RT in both RT groups. Urinary continence can improve for up to 24 months after RP and early RT may interfere with or prolong this return to continence. ${ }^{19}$ In this study, UI was more common in the early RT group at the time of RT and UI rates improved in both groups at years 1,2 , and 3 post-RT. Although the severity of UI was not recorded, only 12 patients in the cohort had an AUS placed. These patients had UI which was severe and bothersome enough to require AUS and there was no difference in the number of AUS surgeries between the 2 RT groups.

Complication rates of RT post-RP vary and depend on the patient and radiation factors, such as timing, dose and technique. ${ }^{23}$ We found that the UI rates were not significantly different in patients receiving early $(24.5 \%)$ or late RT $(23.3 \%)$ at 3 years after RT. In the Southwest Oncology Group (SWOG) 8794 trial, adjuvant RT increased total UI $(6.5 \%$ vs. $2.8 \%)$ compared with the wait-and-see group. ${ }^{3}$ In the 50 men who received salvage RT, Forman and colleagues reported a UI rate of $6 \% .{ }^{24}$ Our UI rates were higher likely due to our subjective definition of UI, as well as the number of patients with unknown status who were not included in the analysis. If we included the unknown patients as continent, 3-year UI rates would have been closer to those reported in the literature $(16.7 \%$ early RT and $14.7 \%$ late RT).

The urethral stricture rate is increased with ART compared with the wait-and-see strategy $(17.8 \%$ vs. $9.5 \%$, respectively). ${ }^{3}$ In the 959 men receiving adjuvant or salvage RT, the reported rates of urethral stricture and $\mathrm{BNC}$ were $1.2 \%$ and $0.8 \%$, respectively. ${ }^{25}$ In our study, BNC developed prior to $\mathrm{RT}$ in more patients than expected $(4.1 \%)$; however, almost all of these resolved despite RT therapy. The high resolution rates of $\mathrm{BNC}$ and urethral stricture demonstrate that treatment of these complications is highly successful. During the first 3 years post-RT, new-onset urethral strictures were rare (4 cases), occurring mostly at 1 and 2 years post-RT. There were no significant differences in the rates of BNC or urethral stricture between early and late RT groups at 3 
years post-RT. Overall, persistent urethral stricture $(0.31 \%)$ and $\mathrm{BNC}(1.1 \%)$ rates at 3 years post-RT correlated with those in the literature.

Intensity-modulated radiotherapy (IMRT) reduces acute toxicity and gastrointestinal side effects, but may not reduce urinary complications because of the difficulty of excluding the bladder neck from the treatment field. ${ }^{15,26}$ Adjuvant RT with high-dose IMRT (>70 Gy) has been shown to have a late UI rate of $18 \%$ and urethral stricture rate of $6 \% .{ }^{17}$ In this study, only a small proportion of the patients received IMRT (96/652, $13 \%$ early RT group and $15 \%$ late RT group) and doses $>66$ Gy were rare. Therefore, the complication rate may be lower than reported with higher radiation doses.

The non-standardized reporting and retrospective nature of data collection of this study is a source of a collection bias. Questionnaires assessing voiding function and incontinence were not routinely used. The degree or bother of the UI (i.e., pad use and weight, questionnaires or urodynamics) was not routinely determined in all patients. The lack of specific assessment of the incontinence and its severity, other than by clinical history, is a limitation in the methodology of this study. However, patient reporting of the presence of UI and whether it was troublesome likely mirrors clinical practice for most urologists and radiation oncologists and gives some understanding to both clinicians and patients as to what can be expected after postoperative RT. UI, BNC and urethral stricture may have been under-reported, especially in patients with mild symptoms, with a bias towards clinically relevant complications being included in the analysis. In addition, if patients had previous UI but their continence status was unknown at 3 years, they were excluded from the analysis, which limits the accuracy of our results.

This study is limited by the definition for early and late RT as they do not strictly match those of adjuvant and salvage RT. However, the results of this study do not demonstrate any impact of timing of postoperative RT on the development of urinary complications and this allows for flexibility in the decision around the optimal timing of the treatment. This study does not take into account how differences in surgical techniques and complications could affect the selection, dosing and toxicity of RT.

\section{Conclusion}

We found that UI was frequently reported after RP and this improved over time regardless of RT timing. Early and late RT showed similar rates of urinary complications (UI, urethral stricture and $\mathrm{BNC}$ ) at 3 years post-RT. This suggests that the timing of radiation does not alter the incidences of these complications after RP. Along with the patient's age, comorbidity, disease and preference for treatment, this added information can be used to make a decision regarding adju- vant versus salvage RT. Further investigation into the timing of postoperative RT and complication rates is underway in 2 phase III trials (RAVES [TROG 08-03] and RADICALS [MRC PR10, NCIC PR13, NCT 00541047]) which may serve to answer these questions in a randomized prospective manner, validate these results, and aid in the decision-making of early versus late RT.

Competing interests: Dr. Sowerby, Dr. Gani, Dr. Yim, Dr. Radomski and Dr. Catton all declare no competing financial or personal interests.

This paper has been peerreviewed.

\section{References}

1. Bolla $M$, van Poppel $H$, Collette $L$, et al. Postoperative radiotherapy after radical prostatectomy: A randomised controlled trial (EORTC trial 22911). Lancet 2005;366:572-8. http://dx.doi.org/10.1016/ S0140-6736(05)67101-2

2. Bolla $M$, van Poppel $H$, Tombal $B$, et al. Postoperative radiotherapy after radical prostatectomy for high-risk prostate cancer: Long-term results of a randomised controlled trial (EORTC trial 22911). Lancet 2012;380:2018-27. http://dx.doi.org/10.1016/S0140-6736(12)61253-7

3. Thompson IM, Tangen CM, Paradelo J, et al. Adjuvant radiotherapy for pathologically advanced prostate cancer: A randomized clinical trial. JAMA 2006;296:2329-35. http://dx.doi.org/10.1001/ jama.296.19.2329

4. Thompson IM, Tangen $C M$, Paradelo J, et al. Adjuvant radiotherapy for pathological T3NOMO prostate cancer significantly reduces risk of metastases and improves survival: Long-term followup of a randomized clinical trial. J Urol 2009;181:956-62. http://dx.doi.org/10.1016/i.juro.2008.11.032

5. Wiegel $T$, Bottke D, Steiner $U$, et al. Phase III postoperative adjuvant radiotherapy after radical prostatectomy compared with radical prostatectomy alone in pT3 prostate cancer with postoperative undetectable prostate-specific antigen: ARO 96-02/AUO AP 09/95. J Clin Oncol 2009;27:2924-30. http://dx.doi. org/10.1200/JC0.2008.18.9563

6. Stephenson AJ, Scardino PT, Kattan MW, et al. Predicting the outcome of salvage radiation therapy for recurrent prostate cancer after radical prostatectomy. J Clin Oncol 2007;25:2035-41. http://dx.doi. org/10.1200/JC0.2006.08.9607

7. Booriian SA, Karnes RJ, Crispen PL, et al. Radiation therapy after radical prostatectomy: Impact on metastasis and survival. J Urol 2009;182:2708-15. http://dx.doi.org/10.1016/i.juro.2009.08.027

8. Trock BJ, Han M, Freedland SJ, et al. Prostate cancer-specific survival following salvage radiotherapy vs observation in men with biochemical recurrence after radical prostatectomy. JAMA 2008;299:2760-9. http://dx.doi.org/10.1001/jama.299.23.2760

9. Song C, Kim YS, Hong JH, et al. Treatment failure and clinical progression after salvage therapy in men with biochemical recurrence after radical prostatectomy: Radiotherapy vs androgen deprivation. BJU Int 2009;106:188-93. http://dx.doi.org/10.1111/i.1464-410X.2009.09136.x

10. Cozzarini C, Bolognesi A, Ceresoli GL, et al. Role of postoperative radiotherapy after pelvic lymphadenectomy and radical retropubic prostatectomy: A single institute experience of 415 patients. Int J Radiat Oncol Biol Phys 2004;59:674-83. http://dx.doi.org/10.1016/i.ijrobp.2003.12.001

11. Hagan M, Zlotecki R, Medina C, et al. Comparison of adjuvant versus salvage radiotherapy policies for postprostatectomy radiotherapy. Int J Radiat Oncol Biol Phys 2004;59:329-40. http://dx.doi. org/10.1016/i.i.irobp.2003.11.038

12. Trabulsi EJ, Valicenti RK, Hanlon AL, et al. A multi-institutional matched-control analysis of adjuvant and salvage postoperative radiation therapy for pT3-4NO prostate cancer. Urology 2008;72:1298-302. http://dx.doi.org/10.1016/i.urology.2008.05.057

13. Ost $P$, De Troyer $B$, Fonteyne VR, et al. A matched control analysis of adjuvant and salvage highdose postoperative intensity-modulated radiotherapy for prostate cancer. Int $J$ Radiat Oncol Biol Phys 2011;80:1316-22. http://dx.doi.org/10.1016/i.i.irobp.2010.04.039

14. Izawa J. Salvage radiotherapy after radical prostatectomy. Can Urol Assoc J 2009;3:245.

15. Cozzarini C, Di Muzio N. Contemporary role of radiation therapy in the adjuvant or salvage setting following radical prostatectomy. Curr Opin Urol 2011;21:206-10. http://dx.doi.org/10.1097/ MOU.0b013e3283449e06 
Sowerby et al.

16. Gabka CJ, Benhaim P, Mathes SJ, et al. An experimental model to determine the effect of irradiated tissue on neutrophil function. Plast Reconstr Surg 1995;96:1676-88. http://dx.doi.org/10.1097/00006534199512000-00023

17. Ost $P$, Fonteyne $V$, Villeirs $G$, et al. Adjuvant high-dose intensity-modulated radiotherapy after radical prostatectomy for prostate cancer: Clinical results in 104 patients. Eur Urol 2009;56:669-75. http:// dx.doi.org/10.1016/j.eururo.2009.05.041

18. Jani AB, Kao J. Postprostatectomy adjuvant versus salvage radiotherapy. Cancer 2005;103:1833-42. http://dx.doi.org/10.1002/cncr.20949

19. Petroski RA, Warlick WB, Herring J, et al. External beam radiation therapy after radical prostatectomy: Efficacy and impact on urinary continence. Prostate Cancer Prostatic Dis 2004;7:170-7. http://dx.doi. org/10.1038/si.pcan.4500718

20. Cox JD, Gallagher MJ, Hammond EH, et al. Consensus statements on radiation therapy of prostate cancer: Guidelines for prostate re-biopsy after radiation and for radiation therapy with rising prostate-specific antigen levels after radical prostatectomy. J Clin Oncol 1999;17:1155.

21. Heidenreich A, Aus G, Bolla M, et al. EAU guidelines on prostate cancer. Eur Urol 2008;53:68-80. http:// dx.doi.org/10.1016/i.eururo.2007.09.002

22. Marks LB, Carroll PR, Dugan TC, et al. The response of the urinary bladder, urethra, and ureter to radiation and chemotherapy. Int J Radiat Oncol Biol Phys 1995;31:1257-80. http://dx.doi.org/10.1016/03603016(94)00431-J
23. Jereczek-Fossa BA, Zerini D, Fodor C, et al. Correlation between acute and late toxicity in 973 prostate cancer patients treated with three-dimensional conformal external beam radiotherapy. Int J Radiat Oncol Biol Phys 2010;78:26-34. http://dx.doi.org/10.1016/i.jirobp.2009.07.1742

24. Forman JD, Duclos $M$, Shamsa F, et al. Predicting the need for adjuvant systemic therapy in patients receiving postprostatectomy irradiation. Urology 1996;47:382-6. http://dx.doi.org/10.1016/S00904295(99)80457-9

25. Feng M, Hanlon AL, Pisansky TM, et al. Predictive factors for late genitourinary and gastrointestinal toxicity in patients with prostate cancer treated with adjuvant or salvage radiotherapy. Int I Radiat Oncol Biol Phys 2007:68:1417-23. http://dx.doi.org/10.1016/i.i.irobp.2007.01.049

26. Alongi F, Fiorino C, Cozzarini C, et al. IMRT significantly reduces acute toxicity of whole-pelvis irradiation in patients treated with post-operative adjuvant or salvage radiotherapy after radical prostatectomy. Radiother and Oncol 2009;93:207-12. http://dx.doi.org/10.1016/i.radonc.2009.08.042

Correspondence: Dr. Sidney B. Radomski, Toronto Western Hospital, Main Pavilion, 8th Floor Rm. 304, 399 Bathurst St., Toronto, ON M5T 2S8; fax: 416-603-5391; sidney.radomski@uhn.ca 